\title{
Association of increased Visfatin/PBEF/NAMPT circulating concentrations and gene expression levels in peripheral blood cells with lipid metabolism and fatty liver in human morbid obesity
}

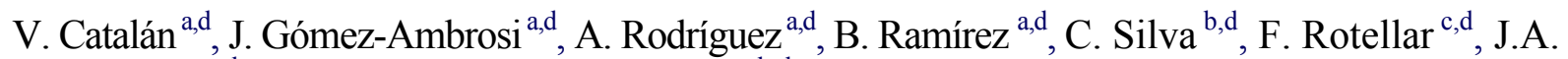 \\ Cienfuegos ${ }^{c, d}$, J. Salvador b,d, G. Frühbeck ${ }^{\text {a,b,d } *}$
}

\begin{abstract}
${ }^{a}$ Metabolic Research Laboratory, Clínica Universitaria de Navarra, University of Navarra, Pamplona, Spain

${ }^{\mathrm{b}}$ Department of Endocrinology, Clínica Universitaria de Navarra, University of Navarra, Pamplona, Spain

${ }^{\mathrm{c}}$ Department of Surgery, Clínica Universitaria de Navarra, University of Navarra, Pamplona, Spain

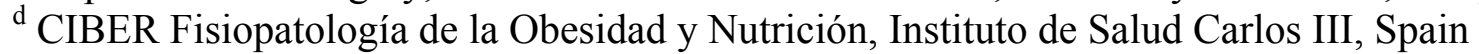

\begin{abstract}
Background and aims: Nicotinamide phosphoribosyltransferase (NAMPT) is an adipokine with physiological effects on the control of glucose homeostasis as well as potentially involved in inflammation. The association of circulating NAMPT concentrations with obesity has not been clearly established. The aim of the present work was to evaluate the effect of obesity on circulating concentrations and gene expression levels of NAMPT in human peripheral blood cells (PBCs) as well as its involvement in inflammation, glucose and lipid metabolism.
\end{abstract}

Methods and results: Forty-four serum samples obtained from 14 lean and 30 obese volunteers were used to analyse the circulating concentrations of NAMPT. In addition, PBC, omental adipose tissue (OM) and liver biopsy samples obtained from a subgroup of subjects were used to determine transcript levels of NAMPT by Real-time PCR. Glucose and lipid profile as well as several inflammatory factors and hepatic enzymes were analysed. NAMPT circulating concentrations $(\mathrm{P}<0.01)$ and gene expression levels in PBC $(\mathrm{P}<0.05)$ were significantly increased in obese patients as compared to lean subjects. Total-cholesterol ( $\mathrm{P}$ $=0.016)$, HDL-cholesterol $(\mathrm{P}=0.036)$ and triglycerides $(\mathrm{P}=0.050)$ were significant and independent determinants of circulating concentrations of NAMPT $(\mathrm{P}<0.01)$. Moreover, a positive correlation $(\mathrm{P}<$ 0.01 ) was found with the hepatic enzymes alanine aminotransferase, aspartate aminotransferase, and $\mathrm{y}-$ glutamyltransferase after BMI adjustment.

Conclusion: Our work shows that NAMPT circulating concentrations and mRNA expression levels in PBC are increased in obese patients and that plasma NAMPT levels are related to inflammation, lipid metabolism and hepatic enzymes suggesting a potential involvement in fatty liver disease and in the obesity-associated inflammatory state.

*Corresponding author at: Department of Endocrinology, Clínica Universitaria de Navarra, University of Navarra, Avda. Pío XII, 36, 31008 Pamplona, Spain. Tel.: p34 948 2554 00x4484; fax: p34 9482965 00. E-mail address: gfruhbeck@,unav.es (G.

Frühbeck). 


\section{INTRODUCTION}

The incidence of obesity has dramatically increased in the last decades with excess adiposity being associated with the increased risk of type 2 diabetes mellitus (T2DM) [1], cardiovascular diseases [2], and negative effects on liver function [3]. Adipose tissue contributes to regulating whole-body metabolism as well as inflammatory and immune responses through the direct effects of adipokines [4-6].

In the past years, a rate-limiting component of the mammalian NAD biosynthesis pathway from nicotinamide called nicotinamide phosphoribosyltransferase (NAMPT) has emerged as a new adipokine [7-10]. NAMPT was first identified as pre-B cell colony-enhancing factor (PBEF), a highly conserved $52 \mathrm{kDa}$ cytokine-like protein that enhances the maturation of B cell precursors in the presence of interleukin (IL)-7 and stem cell factor [11] and inhibits the apoptosis of neutrophils [12]. NAMPT, also named visfatin, has been also identified as a visceral fatderived adipokine with insulin-mimetic effects by binding to and activating the insulin receptor [13]. However, the physiological relevance of NAMPT remains controversial [13-18]. Although increased expression of NAMPT by visceral fat has been described [13], other studies failed to confirm the original finding and no differences were detected between both depots [14,19]. Moreover, whereas some investigators have reported higher plasma visfatin in individuals with obesity [14,20] and T2DM [21,22], other studies have shown opposite findings [23 25]. Revollo et al. [16] were unable to reproduce the insulin-mimetic activity of this protein, but a significant physiological role in the regulation of b-cell function through the NAD biosynthetic activity was detected, suggesting an important role of NAMPT in the control of glucose metabolism.

Obesity is closely linked to systemic inflammation [26]. A new functional link between NAD metabolism and inflammation has been reported, suggesting a potential role for NAD-dependent enzymes in the regulation of proinflammatory cytokine production [27]. Furthermore, NAMPT was found to be predominantly produced and released by the adipose tissue-derived macrophages [28]. The reported association between NAMPT and inflammatory markers raises the possibility of inflammatory properties of NAMPT [29-32]. NAMPT has been shown to upregulate IL-6, IL$1 \mathrm{~b}$ and tumour necrosis factor (TNF) a in human monocytes [30]. It has been proposed that inflammation in adipose tissue in the obese state is a response to hypoxia in enlarged adipocytes distant from the vasculature [33]. In this sense, obesity has been associated with the formation of hypoxic areas within the fat tissue [34,35] with hypoxia-inducible factor-1-a (HIF1 a) being recognised as an important and well-characterized key regulator of the adaptive response to low oxygen tension [36]. Although the inflammatory response is mainly evident in the visceral adipose tissue in the presence of obesity, recent studies indicate that circulating mononuclear cells obtained from obese individuals are in a pro-inflammatory state, being also involved in the increased concentrations of inflammatory cytokines [37-40].

In this study we assessed the influence of obesity on circulating concentrations of NAMPT as well as its gene expression levels in human peripheral blood cells, a type of cells easily accessible and representative of the obesity-associated low-grade chronic inflammation. We also assessed the possible relevance of NAMPT in inflammation, glucose and lipid metabolism in a well-characterized group of subjects including lean and obese patients. 


\section{METHODS}

For detailed Research Design and Methods see Supplementary Research Design and Methods.

\section{Patient selection}

In order to analyse the effects of obesity on plasma NAMPT concentrations, 44 volunteers [14 lean (LN) and 30 obese (OB)] were recruited from healthy individuals and patients attending the Departments of Endocrinology and Surgery of the Clínica Universidad de Navarra. Patients were classified as obese according to both body mass index (BMI $>30 \mathrm{~kg} / \mathrm{m}^{2}$ ) and body fat percentage ( $\mathrm{BF}>25 \%$ for males and $\mathrm{BF}>35 \%$ for females). BMI was calculated as weight in kilograms divided by the square of height in meters and body fat was estimated by air-displacementplethysmography (Bod-Pod ${ }^{\circ}$, Life Measurements, Concord, CA) [41]. Waist-to-hip ratio (WHR) was measured as the ratio between the circumference of the waist (at a midway level between the margin of the lowest rib and the iliac crest) and the hip (at the widest trochanters). Normoglycaemia (NG) and T2DM are defined following the criteria of the Expert Committee on the Diagnosis and Classification of Diabetes Mellitus based on both fasting plasma glucose concentrations and $2 \mathrm{~h}$ after an oral glucose tolerance test (OGTT) [42]. T2DM subjects were not on insulin therapy or on medication likely to influence endogenous insulin levels.

In addition, gene expression levels of NAMPT and HIF1 « in peripheral blood cells (PBC) $(\mathrm{n}=$ $26)$, omental adipose tissue $(n=23)$ and hepatic biopsies $(n=11)$ were assessed in a subgroup of subjects. While an intraoperative liver biopsy was performed in the obese patients undergoing bariatric surgery to obtain a histological diagnosis of the liver state, this procedure is not clinically justified in lean subjects.

The study was approved, from an ethical and scientific standpoint, by the Hospital's Ethical Committee (Comitéde Ética de la Investigación de la Universidad de Navarra) responsible for research and the written informed consent of participants was obtained.

\section{Blood assays}

Biochemical assays of subjects included in the study were measured as previously described [43]. Intra- and interassay coefficients of variation were 5.0 and $4.5 \%$, respectively. NAMPT levels were assessed using a commercially available ELISA kit (ALPCO Diagnostics, Salem, NH, USA) according to the manufacturer's instructions, being the intra- and inter-assay coefficients of variation of 4.3 and $7.6 \%$, respectively.

RNA extraction and real-time PCR

The transcript levels for NAMPT and HIF1-« were quantified by Real-Time PCR (7300 Real-Time PCR System, Applied Biosystem, Foster City, CA, USA) as previously described [43]. Primers and probes (Supplementary Table 1) were designed using the software Primer Express 2.0 (Applied Biosystems). 
Data are presented as mean \pm standard deviation (SD). Differences between the LN and OB groups were assessed by two-tailed unpaired Student's t-test. Gene expression levels were logarithmically transformed because of their non-normal distribution. For $\mathrm{n}$ values below 10 per group, the nonparametric Kruskal-Wallis test, followed by U Mann-Whitney's pairwise comparisons were applied. The normal distribution of the other variables was adequate for the use of parametric tests. Pearson's correlation coefficients ( $r$ ) were used to analyse the association between variables. Multivariate linear regression analyses were conducted for the dependent variable NAMPT, including the parameters which showed a significant correlation with these markers as independent variables. The calculations were performed using the SPSS/Windows version 15.0 statistical package (SPSS, Chicago, IL). A P value $<0.05$ was considered statistically significant.

\section{RESULTS}

Patient characteristics and metabolic profile

The biochemical and hormonal characteristics of the subjects included in the study regarding the circulating concentrations and gene expression levels are shown in Tables 1 and 2, respectively. As expected, obese patients showed significantly higher BF $(\mathrm{P}<0.0001)$ and WHR $(\mathrm{P}$ $<0.0001)$ as compared to the LN volunteers. Obese patients exhibited lower insulin sensitivity than LN individuals as evidenced by the higher HOMA $(\mathrm{P}<0.01)$ and lower QUICKI $(\mathrm{P}<0.001)$ indices. Circulating concentrations of triglycerides were significantly increased in OB patients $(\mathrm{P}<$ $0.05)$, whereas HDL-cholesterol was reduced $(\mathrm{P}<0.01)$. Circulating levels of the inflammatory markers CRP $(\mathrm{P}<0.05)$, fibrinogen $(\mathrm{P}<0.001)$, and $\mathrm{vWF}(\mathrm{P}<0.05)$ were increased in $\mathrm{OB}$ patients compared to LN subjects as the concentration of the hepatic enzyme ALT $(\mathrm{P}<0.001)$, As expected, the high $\mathrm{BF}$ values were accompanied by high leptin concentrations $(\mathrm{P}<0.0001)$.

Circulating and gene expression levels of NAMPT in PBC are elevated in human obesity

Circulating concentrations of NAMPT were significantly increased $(\mathrm{P}=0.002)$ in $\mathrm{OB}$ patients compared to LN subjects (Fig.1A). Moreover, a positive correlation was found between circulating NAMPT levels and both BMI $(\mathrm{r}=0.31 ; \mathrm{P}=0.044)$ and WHR $(\mathrm{r}=0.47 ; \mathrm{P}=0.012)$ (Table 3$)$.

Real-time PCR analysis indicated that the mRNA expression of NAMPT in PBC followed the same pattern, being significantly higher $(\mathrm{P}=0.013)$ in $\mathrm{OB}$ subjects compared to LN controls (Fig. 1B). A positive correlation between NAMPT gene expression levels and BMI $(\mathrm{r}=0.50 ; \mathrm{P}=$ $0.009), \mathrm{BF}(\mathrm{r}=0.48 ; \mathrm{P}=0.014)$ and $\mathrm{WHR}(\mathrm{r}=0.43 ; \mathrm{P}=0.032)$ was detected (Table 3$)$. No differences $(\mathrm{P}=0.387)$ in omental adipose tissue gene expression levels of NAMPT between LN and OB subjects were observed (Fig. 1C). Although a tendency towards a decreased NAMPT gene expression was evident in liver biopsies of obese T2DM patients compared to obese NG subjects, it did not reach statistical significance (NG: $1.00 \pm 0.28$, T2DM: $0.66 \pm 0.41$ arbitrary units; $\mathrm{P}=0.330$ ).

Gene expression levels of NAMPT in PBC of obese subjects were significantly increased compared to omental adipose tissue $(\mathrm{P}=0.029)$ or liver expression $(\mathrm{P}=0.029)$ (Fig. 1D). Moreover, no significant associations between NAMPT circulating levels and gene expression in $\mathrm{PBC}(\mathrm{r}=0.24 ; \mathrm{P}=$ $0.244)$, omental adipose tissue $(\mathrm{r}=0.03 ; \mathrm{P}=0.881)$ or liver $(\mathrm{r}=0.43 ; \mathrm{P}=0.212)$ were detected. 
NAMPT levels and glucose metabolism

NAMPT circulating levels were positively correlated with glucose concentrations $(\mathrm{r}=0.33 ; \mathrm{P}=$ 0.049) although the association was lost after BMI adjustment (Table 3). Analogously, a positive correlation between gene expression levels of NAMPT in PBC and insulin concentrations $(\mathrm{r}=0.54$; $\mathrm{P}=0.014)$ as well as the HOMA index $(\mathrm{r}=0.59 ; \mathrm{P}=0.016)$ was observed. However, these associations were also lost after BMI adjustment (Table 3).

NAMPT levels and hepatic profile

A significant positive correlation $(\mathrm{P}<0.01)$ was found between plasma NAMPT concentrations and ALT, AST and y-GT levels (Table 3). Moreover, the association of circulating levels of NAMPT with BMI or WHR was lost after adjusting for ALT, AST, ALP or y-GT (data not shown). NAMPT gene expression levels in PBC were positively correlated $(\mathrm{P}<0.05)$ with ALP and $y-G T$ with the correlation being maintained after BMI adjustment (Table 3). Furthermore, hepatic gene expression levels of NAMPT were positively associated $(\mathrm{P}<0.01)$ with AST and ALT concentrations after BMI adjustment.

\section{NAMPT levels and inflammation}

Inflammation may be a possible mechanism whereby hepatic enzyme concentrations are elevated. Circulating NAMPT and gene expression levels in PBC were positively correlated with the inflammatory markers CRP $(\mathrm{P}<0.05)$, fibrinogen $(\mathrm{P}<0.05)$ and $\mathrm{vWF}(\mathrm{P}<0.05)$, although the associations were lost after BMI adjustment (Table 3 ). In addition, a positive correlation between NAMPT and HIF1 «mRNA expression levels in PBC was found $(\mathrm{r}=0.51 ; \mathrm{P}=0.011)$, remaining statistically significant after BMI adjustment $(\mathrm{r}=0.45 ; \mathrm{P}=0.030)$. No association between gene expression levels of NAMPT and HIF1-« in omental adipose tissue as well as liver were observed (data not shown).

NAMPT levels and lipid metabolism

Circulating concentrations of NAMPT were positively correlated with triglycerides $(\mathrm{P}<0.05)$, total-cholesterol $(\mathrm{P}<0.001)$ and LDL-cholesterol $(\mathrm{P}<0.001)$ (Table 3). In order to better characterise the relationship between circulating levels of NAMPT and lipid metabolism as well as to find out which variables better predict it, multivariate linear regression analysis was performed, including CRP concentrations in the model due to their significant association with the dependent variable NAMPT. The model that best predicted NAMPT levels explained 35.6\% of its variability (Table 4). Total-cholesterol (P Z 0.016), HDLcholesterol (P Z 0.036) and triglycerides ( $\mathrm{P} Z \mathrm{Z}$ 0.050) were significant independent determinants of NAMPT, suggesting a more pronounced influence of lipid metabolism on NAMPT circulating levels than inflammation. NAMPT gene expression levels of $\mathrm{PBC}$ were positively correlated with triglyceride concentrations $(\mathrm{r}=0.49$; $\mathrm{P}$ $=0.033)$ with the association being maintained after BMI adjustment $(\mathrm{r}=0.44 ; \mathrm{P}=0.043)$ (Table $3)$. 


\section{DISCUSSION}

Adipose tissue acts as an active paracrine and endocrine organ, secreting a pleiad of adipokines that participate in diverse metabolic processes. In this sense, NAMPT has been described as an adipokine with complex biological effects although its mechanisms of action are still incompletely understood. The main findings of this study are: (1) that NAMPT circulating concentrations and mRNA expression levels in PBC are increased in obese patients, and (2) that plasma NAMPT levels are related to inflammation, lipid metabolism and hepatic enzymes suggesting a potential involvement in fatty liver disease.

We detected increased circulating concentrations of NAMPT in obese patients as well as a positive association with BMI and WHR, which is in agreement with previous [14,20], but not all [24,25] data. In this sense, qualitative and quantitative discrepancies in the detection of NAMPT by EIA, RIA and ELISA immunoassays have been described, trying to explain the conflicting observations as regards the putative alterations of circulating NAMPT in human obesity. It has been suggested that ELISA is the most specific and applicable assay for NAMPT detection in human serum [44]. We measured circulating NAMPT by a commercial ELISA assay that recognised the full-length protein, since the importance of the detection of full-length NAMPT rather than the carboxy-terminal NAMPT to clarify the physiological role of this protein has been recently described [45]. To date, most clinical studies have used a competitive assay that recognizes the carboxy-terminal of the visfatin protein. In these studies, the reported concentrations of circulating visfatin have shown marked variability, ranging from 1 to $50 \mathrm{ng} / \mathrm{ml}$. Indeed, it has been suggested that the C-terminal assay measures recombinant full-length human visfatin in a range around 100-200-fold lower than the true values [45]. These data support recent suggestions that this assay is compromised by interference with a high molecular weight compound. The dimerization of visfatin may have functional implications, particularly in regard to its NADp biosynthetic enzyme function that has been shown to depend on dimerization and to be active also in the extracellular space [44]. Our study provides evidence that obese patients exhibit upregulated NAMPT mRNA expression levels in PBC, contrasting with previously published results where a negative or no correlation with BMI was detected $[39,46]$. However, a positive correlation has been shown with waist circumference measures, a better indicator of visceral obesity [46]. The lack of association between the circulating concentrations and the mRNA expression levels of NAMPT in PBC, omental adipose tissue and liver suggests that other organs or cell types, including bone marrow or muscle [11], may be contributing to the increased NAMPT levels in obesity. Noteworthy, accumulation of PBC at sites of inflammation may play a significant role in the secretion of NAMPT. Further research will provide more insight into the still unclear topic of the main source of increased NAMPT in human obesity.

Although a potential relationship between NAMPT and glucose homeostasis has been previously suggested [13,21], no significant correlation between NAMPT concentrations and variables of insulin sensitivity, including fasting insulin and glucose concentrations have been described by other authors $[14,24,25]$. Furthermore, Revollo et al. reported that they were unable to reproduce the insulin-mimetic activity of this protein, stimulation of glucose uptake, and the glucose-lowering effect in vivo [16]. In line with these studies, our data suggest that NAMPT may not play a major role in the control of glucose homeostasis given the lack of association with plasma glucose and insulin after BMI adjustment.

Dyslipidaemia is an important component of the metabolic syndrome. We have found a positive association between circulating NAMPT levels and triglycerides, totaland LDL-cholesterol suggesting a role of NAMPT in lipid homeostasis. In line with this observation, a role of NAMPT in lipid metabolism has been put forward due to its strong correlation with serum triglycerides independently of age and BF [47,48] and with total- and LDL-cholesterol [49]. Contrarily, plasma NAMPT levels have been associated with a high HDL-cholesterol/low triglyceride plasma lipid 
profile [50]. The different results obtained might be explained by the non-fasting samples used in the latter study. Recent work has shown that NAMPT may be connected with the lipid profile through NAD metabolism given its extra- and intra-cellular NAD biosynthetic properties. It has been shown that nicotinic acid increases HDL-cholesterol and also reduces triglyceride-rich lipoproteins [51]. In our study, the elevated concentrations of NAMPT may reflect a compensatory mechanism in order to reduce triglyceride accumulation. However, the role of NAMPT in lipid metabolism remains to be fully disentangled.

The present study shows that increased NAMPT levels are accompanied by high concentrations of ALT, AST and g-GT, which are commonly increased in obese patients in relation to fatty liver disease. The elevated concentrations of hepatic enzymes exhibited by the obese patients may be influencing NAMPT concentrations or, conversely, NAMPT may be exerting an effect on the concentrations of the liver enzymes. In this sense, the protective role of NAMPT in non-alcoholic fatty liver disease (NAFLD) [52] and its involvement in portal inflammation in NAFLD patients has been recently reported [53]. NAD is a coenzyme with important roles in a variety of biological processes in part through the activation of sirtuin-1, a NAD(b)-dependent histone deacetylase involved in the control of metabolic processes [54]. It has been recently suggested that an increase of sirtuin-1 exerts protective effects against the development of NAFLD in rats [55] and alcoholic liver steatosis in mice [56], suppressing the expression of genes encoding lipogenic enzymes and preventing lipid accumulation in the liver.

Chronic low-grade inflammation constitutes a mediator in the development of obesity-related diseases. The association of NAMPT with other inflammatory markers, such as MCP-1 and PAI-1 antigen [57] together with its increased production and release by macrophages [28,58] supports its pro-inflammatory properties [59]. Furthermore, a strong association between NAMPT and TNF-a and IL-8 in PBC has been described [39,46,58]. However, Varma et al. [19] found a negative association between NAMPT and CD68 as well as TNF-a in adipose tissue, suggesting an antiinflammatory role for NAMPT. We herein provide evidence for an association of NAMPT with CRP and fibrinogen, well-established markers of chronic inflammation. These acute phase proteins are released by the liver suggesting a hepatic response to inflammation rather than a systemic inflammatory process as indicated by the pattern of cytokine release. In agreement with previous results performed in 3T3-L1 adipocytes and MCF-7 cells [60,61], we found that gene expression of NAMPT in PBC in vivo, was positively correlated with HIF1-« mRNA expression in humans, a wellcharacterized key regulator of the adaptive response to low oxygen tension present in the obesity-associated inflammation state $[34,62,63]$. Taken together, these associations may contribute to the role of NAMPT in the obesity-associated inflammatory state, which takes place in the obesity-related cardiovascular derangements.

In summary, we show that NAMPT circulating concentrations and gene expression levels in human PBC are increased in obese patients. Moreover, the association of NAMPT with triglycerides, hepatic enzymes and proinflammatory markers suggest an involvement in the dyslipidemia, fatty liver disease and low-grade chronic inflammation accompanying obesity. Further studies to better understand the complex implication of this adipokine in obesity and obesity-associated cardiovascular diseases are warranted.

\section{DISCLOSURE STATEMENT}

The authors have nothing to disclose. 


\section{FUNDING}

This work was supported by FIS, PI061458 and PI06/90288 from the Spanish Instituto de Salud Carlos III, Ministerio de Sanidad y Consumo, by the Department of Health (20/2005 and 3/2006), by the Department of Education of the Gobierno de Navarra of Spain (res/2008) and by Fundación Mutua Madrileña.

\section{ACKNOWLEDGEMENTS}

The authors gratefully acknowledge the valuable collaboration of all the members of the Multidisciplinary Obesity Team. CIBER de Fisiopatología de la Obesidad y Nutrición (CIBEROBN) is an initiative of the Instituto de Salud Carlos III, Spain.

\section{REFERENCES}

[1] Guilherme A, Virbasius JV, Puri V, Czech MP. Adipocyte dysfunctions linking obesity to insulin resistance and type 2 diabetes. Nat Rev Mol Cell Biol 2008;9:367-77.

[2] Van Gaal LF, Mertens IL, De Block CE. Mechanisms linking obesity with cardiovascular disease. Nature 2006;444:875-80.

[3] Tilg H, Hotamisligil GS. Nonalcoholic fatty liver disease: cytokine-adipokine interplay and regulation of insulin resistance. Gastroenterology 2006;131:934-45.

[4] Frühbeck G, Gómez-Ambrosi J. Rationale for the existence of additional adipostatic hormones. FASEB J 2001;15:1996-2006.

[5] Fortuño A, Rodríguez A, Gómez-Ambrosi J, Frühbeck G, Diez J. Adipose tissue as an endocrine organ: role of leptin and adiponectin in the pathogenesis of cardiovascular diseases. J Physiol Biochem 2003;59:51-60.

[6] Badman MK, Flier JS. The adipocyte as an active participant in energy balance and metabolism. Gastroenterology 2007;132: 2103-15.

[7] Rongvaux A, Shea RJ, Mulks MH, Gigot D, Urbain J, Leo O, et al. Pre-B-cell colony-enhancing factor, whose expression is up-regulated in activated lymphocytes, is a nicotinamide phosphoribosyltransferase, a cytosolic enzyme involved in NAD biosynthesis. Eur J Immunol 2002;32:3225-34.

[8] Revollo JR, Grimm AA, Imai S. The NAD biosynthesis pathway mediated by nicotinamide phosphoribosyltransferase regulates Sir2 activity in mammalian cells. J Biol Chem 2004;279: 50754-6.

[9] Sommer G, Garten A, Petzold S, Beck-Sickinger AG, Bluher M, Stumvoll M, et al. Visfatin/PBEF/Nampt: structure, regulation and potential function of a novel adipokine. Clin Sci 2008;115: 13-23. 
[10] Revollo JR, Grimm AA, Imai S. The regulation of nicotinamide adenine dinucleotide biosynthesis by Nampt/PBEF/visfatin in mammals. Curr Opin Gastroenterol 2007;23:164-70.

[11] Samal B, Sun Y, Stearns G, Xie C, Suggs S, McNiece I. Cloning and characterization of the cDNA encoding a novel human preB-cell colony-enhancing factor. Mol Cell Biol 1994;14:1431-7.

[12] Jia SH, Li Y, Parodo J, Kapus A, Fan L, Rotstein OD, et al. Pre-B cell colony-enhancing factor inhibits neutrophil apoptosis in experimental inflammation and clinical sepsis. J Clin Invest 2004;113:1318-27.

[13] Fukuhara A, Matsuda M, Nishizawa M, Segawa K, Tanaka M, Kishimoto K, et al. Visfatin: a protein secreted by visceral fat that mimics the effects of insulin. Science 2005;307:426-30.

[14] BerndtJ, Kloting N, Kralisch S, Kovacs P, FasshauerM, Schon MR, et al. Plasma visfatin concentrations and fat depot-specific mRNA expression in humans. Diabetes 2005;54:2911-6.

[15] Arner P. Visfatin-a true or false trail to type 2 diabetes mellitus. J Clin Endocrinol Metab 2006;91:28-30.

[16] Revollo JR, Korner A, Mills KF, Satoh A, Wang T, Garten A, et al. Nampt/PBEF/Visfatin regulates insulin secretion in 0 cells as a systemic NAD biosynthetic enzyme. Cell Metab 2007;6:363-75.

[17] Fukuhara A, Matsuda M, Nishizawa M, Segawa K, Tanaka M, Kishimoto K, et al. Retraction. Science 2007;318:565.

[18] de Luis DA, Sagrado MG, Aller R, Conde R, Izaola O. Circulating visfatin in obese non-diabetic patients in relation to cardiovascular risk factors, insulin resistance, and adipocytokines: A contradictory piece of the puzzle. Nutrition, in press. doi: 10.1016/j.nutr.2008.11.019.

[19] Varma V, Yao-Borengasser A, Rasouli N, Bodles AM, Phanavanh B, Lee MJ, et al. Human visfatin expression: relationship to insulin sensitivity, intramyocellular lipids, and inflammation. $\mathrm{J}$ Clin Endocrinol Metab 2007;92:666-72.

[20] Haider DG, Schindler K, Schaller G, Prager G, Wolzt M, Ludvik B. Increased plasma visfatin concentrations in morbidly obese subjects are reduced after gastric banding. J Clin Endocrinol Metab 2006;91:1578-81.

[21] Chen MP, Chung FM, Chang DM, Tsai JC, Huang HF, Shin SJ, et al. Elevated plasma level of visfatin/pre-B cell colonyenhancing factor in patients with type 2 diabetes mellitus. J Clin Endocrinol Metab 2006;91:295-9.

[22] López-Bermejo A, Chico-Julia B, Fernández-Balsells M, Recasens M, Esteve E, Casamitjana R, et al. Serum visfatin increases with progressive R-cell deterioration. Diabetes 2006;55:2871-5.

[23] Hammarstedt A, Pihlajamaki J, Rotter Sopasakis V, Gogg S, Jansson PA, Laakso M, et al. Visfatin is an adipokine, but it is not regulated by thiazolidinediones. J Clin Endocrinol Metab 2006;91:1181-4.

[24] Pagano C, Pilon C, Olivieri M, Mason P, Fabris R, Serra R, et al. Reduced plasma visfatin/pre-B cell colony-enhancing factor in obesity is not related to insulin resistance in humans. J Clin Endocrinol Metab 2006;91:3165-70. 
[25] Ingelsson E, Larson MG, Fox CS, Yin X, Wang TJ, Lipinska I, et al. Clinical correlates of circulating visfatin levels in a community-based sample. Diabetes Care 2007;30:1278-80.

[26] Tilg H, Moschen AR. Inflammatory mechanisms in the regulation of insulin resistance. Mol Med 2008;14:222-31.

[27] Busso N, Karababa M, Nobile M, Rolaz A, Van Gool F, Galli M, et al. Pharmacological inhibition of nicotinamide phosphoribosyltransferase/visfatin enzymatic activity identifies a new inflammatory pathway linked to NAD. PLoS ONE 2008;3:e2267.

[28] Curat CA, Wegner V, Sengenes C, Miranville A, Tonus C, Busse R, et al. Macrophages in human visceral adipose tissue: increased accumulation in obesity and a source of resistin and visfatin. Diabetologia 2006;49:744-7.

[29] 29] Oki K, Yamane K, Kamei N, Nojima H, Kohno N. Circulating visfatin level is correlated with inflammation, but not with insulin resistance. Clin Endocrinol 2007;67:796-800.

[30] Moschen AR, Kaser A, Enrich B, Mosheimer B, Theurl M, Niederegger H, et al. Visfatin, an adipocytokine with proinflammatory and immunomodulating properties. $\mathrm{J}$ Immunol 2007; 178:1748-58.

[31] Seo JA, Jang ES, Kim BG, Ryu OH, Kim HY, Lee KW, et al. Plasma visfatin levels are positively associated with circulating interleukin-6 in apparently healthy Korean women. Diabetes Res Clin Pract 2008;79:108-11.

[32] Tilg H, Moschen AR. Role of adiponectin and PBEF/visfatin as regulators of inflammation: involvement in obesity-associated diseases. Clin Sci 2008;114:275-88.

[33] Trayhurn P, Wang B, Wood IS. Hypoxia in adipose tissue: a basis for the dysregulation of tissue function in obesity? Br J Nutr 2008;100:227-35.

[34] Hosogai N, Fukuhara A, Oshima K, Miyata Y, Tanaka S, Segawa K, et al. Adipose tissue hypoxia in obesity and its impact on adipocytokine dysregulation. Diabetes 2007;56: 901-11.

[35] Rausch ME, Weisberg S, Vardhana P, Tortoriello DV. Obesity in C57BL/6J mice is characterized by adipose tissue hypoxia and cytotoxic T-cell infiltration. Int J Obes 2008;32:451-63.

[36] Ke Q, Costa M. Hypoxia-inducible factor-1 (HIF-1). Mol Pharmacol 2006;70:1469-80.

[37] Ghanim H, Aljada A, Hofmeyer D, Syed T, Mohanty P, Dandona P. Circulating mononuclear cells in the obese are in a proinflammatory state. Circulation 2004;110:1564-71.

[38] Dandona P, Aljada A, Ghanim H, Mohanty P, Tripathy C, Hofmeyer D, et al. Increased plasma concentration of macrophage migration inhibitory factor (MIF) and MIF mRNA in mononuclear cells in the obese and the suppressive action of metformin. J Clin Endocrinol Metab 2004;89:50437.

[39] Samara A, Pfister M, Marie B, Visvikis-Siest S. Visfatin, lowgrade inflammation and body mass index (BMI). Clin Endocrinol 2008;69:568-74.

[40] Sheu WH, Chang TM, Lee WJ, Ou HC, Wu CM, Tseng LN, et al. Effect of weight loss on proinflammatory state of mononuclear cells in obese women. Obesity 2008;16:1033-8. 
[41] Das SK, Roberts SB, Kehayias JJ, Wang J, Hsu LK, Shikora SA, et al. Body composition assessment in extreme obesity and after massive weight loss induced by gastric bypass surgery. Am J Physiol Endocrinol Metab 2003;284:E1080-8.

[42] Genuth S, Alberti KG, Bennett P, Buse J, Defronzo R, Kahn R, et al. Follow-up report on the diagnosis of diabetes mellitus. Diabetes Care 2003;26:3160-7.

[43] Catalán V, Gómez-Ambrosi J, Rodriguez A, Ramirez B, Silva C, Rotellar F, et al. Increased adipose tissue expression of lipocalin-2 in obesity is related to inflammation and matrix metalloproteinase-2 and metalloproteinase-9 activities in humans. J Mol Med 2009;87:803-13.

[44] Korner A, Garten A, Bluher M, Tauscher R, Kratzsch J, Kiess W. Molecular characteristics of serum visfatin and differential detection by immunoassays. J Clin Endocrinol Metab 2007;92: 4783-91.

[45] Retnakaran R, Youn BS, Liu Y, Hanley AJ, Lee NS, Park JW, et al. Correlation of circulating fulllength visfatin (PBEF/ NAMPT) with metabolic parameters in subjects with and without diabetes: a cross-sectional study. Clin Endocrinol 2008;69:885-93.

[46] Tsiotra PC, Tsigos C, Yfanti E, Anastasiou E, Vikentiou M, Psarra K, et al. Visfatin, TNF-a and IL-6 mRNA expression is increased in mononuclear cells from type 2 diabetic women. Horm Metab Res 2007;39:758-63.

[47] Filippatos TD, Derdemezis CS, Kiortsis DN, Tselepis AD, Elisaf MS. Increased plasma levels of visfatin/pre-B cell colony-enhancing factor in obese and overweight patients with metabolic syndrome. J Endocrinol Invest 2007;30:323-6.

[48] Sun G, Bishop J, Khalili S, Vasdev S, Gill V, Pace D, et al. Serum visfatin concentrations are positively correlated with serum triacylglycerols and down-regulated by overfeeding in healthy young men. Am J Clin Nutr 2007;85:399-404.

[49] Zhong M, Tan HW, Gong HP, Wang SF, Zhang Y, Zhang W. Increased serum visfatin in patients with metabolic syndrome and carotid atherosclerosis. Clin Endocrinol 2008;69:878-84.

[50] Wang P, van Greevenbroek MM, Bouwman FG, Brouwers MC, van der Kallen CJ, Smit E, et al. The circulating PBEF/ NAMPT/visfatin level is associated with a beneficial blood lipid profile. Pflügers Arch 2007;454:971-6.

[51] Chapman MJ, Assmann G, Fruchart JC, Shepherd J, Sirtori C. Raising high-density lipoprotein cholesterol with reduction of cardiovascular risk: the role of nicotinic acid-a position paper developed by the European Consensus Panel on HDL-C. Curr Med Res Opin 2004;20:1253-68.

[52] Jarrar MH, Baranova A, Collantes R, Ranard B, Stepanova M, Bennett C, et al. Adipokines and cytokines in non-alcoholic fatty liver disease. Aliment Pharmacol Ther 2008;27:412-21.

[53] Aller R, de Luis DA, Izaola O, Sagrado MG, Conde R, Velasco MC, et al. Influence of Visfatin on histopathological changes of non-alcoholic fatty liver disease. Dig Dis Sci 2008; 54:1772-7.

[54]Hara N, Yamada K, Shibata T, Osago H, Hashimoto T, Tsuchiya M. Elevation of cellular NAD levels by nicotinic acid and involvement of nicotinic acid phosphoribosyltransferase in human cells. J Biol Chem 2007;282:24574-82. 
[55]Deng XQ, Chen LL, Li NX. The expression of SIRT1 in nonalcoholic fatty liver disease induced by high-fat diet in rats. Liver Int 2007;27:708-15.

[56] You M, Cao Q, Liang X, Ajmo JM, Ness GC. Mammalian sirtuin 1 is involved in the protective action of dietary saturated fat against alcoholic fatty liver in mice. J Nutr 2008; 138:497-501.

[57] Malavazos AE, Ermetici F, Cereda E, Coman C, Locati M, Morricone L, et al. Epicardial fat thickness: relationship with plasma visfatin and plasminogen activator inhibitor-1 levels in visceral obesity. Nutr Metab Cardiovasc Dis 2008; 18:523-30.

[58]Dahl TB, Yndestad A, Skjelland M, Oie E, Dahl A, Michelsen A, et al. Increased expression of visfatin in macrophages of human unstable carotid and coronary atherosclerosis: possible role in inflammation and plaque destabilization. Circulation 2007;115:972-80.

[59]Zhang YY, Gottardo L, Thompson R, Powers C, Nolan D, Duffy J, et al. A visfatin promoter polymorphism is associated with low-grade inflammation and type 2 diabetes. Obesity 2006;14:2119-26.

[60]Bae SK, Kim SR, Kim JG, Kim JY, Koo TH, Jang HO, et al. Hypoxic induction of human visfatin gene is directly mediated by hypoxia-inducible factor-1. FEBS Lett 2006;580:4105-13.

[61]Segawa K, Fukuhara A, Hosogai N, Morita K, Okuno Y, Tanaka M, et al. Visfatin in adipocytes is upregulated by hypoxia through HIF1a-dependent mechanism. Biochem Biophys Res Commun 2006;349:875-82.

[62] Wang B, Wood IS, Trayhurn P. Dysregulation of the expression and secretion of inflammation-related adipokines by hypoxia in human adipocytes. Pflügers Arch 2007;455: 479-92.

[63] Ye J, Gao Z, Yin J, He Q. Hypoxia is a potential risk factor for chronic inflammation and adiponectin reduction in adipose tissue of ob/ob and dietary obese mice. Am J Physiol Endocrinol Metab 2007;293:E1118-28. 
Table 1 Anthropometric and biochemical characteristics of subjects included in the study of circulating concentrations.

\begin{tabular}{|l|l|l|l|}
\hline & Lean & Obese & P \\
\hline n (Female, male) & $14(13,1)$ & $30(24,6)$ & \\
\hline Age (years) & $35 \pm 11$ & $40 \pm 11$ & 0.140 \\
\hline BMI (kg/m2) & $20.4 \pm 2.2$ & $46.4 \pm 8.0$ & $<0.0001$ \\
\hline Body fat (\%) & $26.4 \pm 5.9$ & $51.1 \pm 7.2$ & $<0.0001$ \\
\hline WHR & $0.75 \pm 0.04$ & $0.95 \pm 0.07$ & $<0.0001$ \\
\hline Fasting glucose (mmol/l) & $4.8 \pm 0.6$ & $6.2 \pm 2.4$ & 0.010 \\
\hline 2h OGTT glucose (mmol/1) & $6.0 \pm 1.2$ & $9.1 \pm 4.6$ & 0.151 \\
\hline Fasting insulin (pmol/1) & $46.9 \pm 6.3$ & $158.9 \pm 65.8$ & $<0.0001$ \\
\hline 2h OGTT insulin (pmol/l) & $73.5 \pm 11.9$ & $678.3 \pm 357.7$ & $<0.0001$ \\
\hline HOMA & $1.5 \pm 0.9$ & $6.9 \pm 5.2$ & $<0.0001$ \\
\hline QUICKI & $0.374 \pm 0.041$ & $0.301 \pm 0.027$ & $<0.0001$ \\
\hline Triglycerides $(\mathrm{mmol} / \mathrm{l})$ & $0.75 \pm 0.25$ & $1.86 \pm 0.40$ & 0.040 \\
\hline Cholesterol (mmol/l) & $4.64 \pm 0.65$ & $5.00 \pm 0.85$ & 0.181 \\
\hline LDL-cholesterol (mmol/l) & $2.68 \pm 0.72$ & $3.21 \pm 0.77$ & 0.053 \\
\hline HDL-cholesterol (mmol/l) & $1.77 \pm 0.58$ & $1.09 \pm 0.24$ & $<0.001$ \\
\hline Leptin (ng/ml) & $8.5 \pm 4.3$ & $60.9 \pm 26.1$ & $<0.0001$ \\
\hline Uric acid (mmol/l) & $0.23 \pm 0.03$ & $0.33 \pm 0.05$ & $<0.0001$ \\
\hline CRP(mg/l) & $1.1 \pm 0.9$ & $7.3 \pm 5.3$ & $<0.0001$ \\
\hline Fibrinogen (mmol/l) & $2.08 \pm 0.64$ & $3.51 \pm 0.5$ & $<0.0001$ \\
\hline vWF (\%) & $81 \pm 53$ & $127 \pm 49$ & 0.012 \\
\hline Homocysteine ( $\mu \mathrm{mol} / \mathrm{l})$ & $6.3 \pm 1.3$ & $8.2 \pm 3.3$ & 0.057 \\
\hline ALT (UI/l) & $7 \pm 2$ & $32 \pm 18$ & $<0.0001$ \\
\hline AST (UI/l) & $12 \pm 3$ & $17 \pm 6$ & 0.017 \\
\hline ALP (UI/l) & $90 \pm 20$ & $110 \pm 30$ & 0.036 \\
\hline$\gamma$-GT (UI/1) & $10 \pm 6$ & $30 \pm 31$ & 0.007 \\
\hline
\end{tabular}

ALT, alanine aminotransferase; ALP, alkaline phosphatase; AST, aspartate aminotransferase; BMI, body mass index; CRP, C-reactive protein; $\gamma$-GT, $\gamma$-glutamyltransferase; HOMA, homeostatic model assessment; OGTT, oral glucose tolerance test; QUICKI, quantitative insulin sensitivity check index; vWF, von Willebrand factor; WHR, waist-to-hip ratio. Data are mean \pm SD. Differences between groups were analysed by twotailed unpaired Student's t-test. 
Table 2 Anthropometric and biochemical characteristics of subjects included in the gene expression study.

\begin{tabular}{llll} 
& Lean & Obese & P \\
\hline n (Female, male) & $8(8,0)$ & $18(12,6)$ & \\
Age (years) & $33 \pm 13$ & $43 \pm 12$ & 0.069 \\
BMI (kg/m2) & $19.7 \pm 2.0$ & $44.9 \pm 7.9$ & $<0.0001$ \\
Body fat (\%) & $26.5 \pm 4.8$ & $49.3 \pm 8.3$ & $<0.0001$ \\
WHR & $0.74 \pm 0.04$ & $0.95 \pm 0.08$ & $<0.0001$ \\
Fasting glucose (mmol/l) & $4.6 \pm 0.7$ & $6.5 \pm 3.1$ & 0.113 \\
Fasting insulin (pmol/1) & $47.0 \pm 25.2$ & $157.8 \pm 65.5$ & $<0.0001$ \\
HOMA & $1.3 \pm 1.0$ & $8.5 \pm 6.1$ & 0.005 \\
QUICKI & $0.386 \pm 0.044$ & $0.290 \pm 0.023$ & $<0.001$ \\
Triglycerides (mmol/1) & $0.73 \pm 0.27$ & $2.59 \pm 0.59$ & 0.062 \\
Cholesterol (mmol/l) & $4.58 \pm 0.67$ & $5.10 \pm 0.89$ & 0.185 \\
LDL-cholesterol (mmol/l) & $2.22 \pm 0.41$ & $3.06 \pm 0.87$ & 0.032 \\
HDL-cholesterol (mmol/l) & $2.07 \pm 0.65$ & $1.17 \pm 0.31$ & $<0.001$ \\
Leptin (ng/ml) & $8.6 \pm 4.9$ & $57.9 \pm 23.2$ & $<0.0001$ \\
Uric acid (mmol/l) & $0.25 \pm 0.03$ & $0.35 \pm 0.07$ & 0.004 \\
CRP (mg/l) & $0.6 \pm 0.3$ & $7.4 \pm 2.6$ & 0.032 \\
Fibrinogen (mmol/l) & $1.90 \pm 0.15$ & $3.36 \pm 0.53$ & $<0.001$ \\
vWF (\%) & $61 \pm 23$ & $123 \pm 43$ & 0.003 \\
Homocysteine $(\mu \mathrm{mol} / \mathrm{l})$ & $6.6 \pm 1.6$ & $10.2 \pm 3.8$ & 0.028 \\
ALT (UI/l) & $6 \pm 2$ & $33 \pm 20$ & $<0.001$ \\
AST (UI/l) & $12 \pm 7$ & $17 \pm 6$ & 0.145 \\
ALP (UI/l) & $86 \pm 23$ & $101 \pm 25 \pm 12$ & 0.171 \\
$\gamma$-GT (UI/l) & & & \\
\hline
\end{tabular}

ALT, alanine aminotransferase; ALP, alkaline phosphatase; AST, aspartate aminotransferase; BMI, body mass index; CRP, C-reactive protein; $\gamma$-GT, $\gamma$-glutamyltransferase; HOMA, homeostatic model assessment; OGTT, oral glucose tolerance test; QUICKI, quantitative insulin sensitivity check index; vWF, von Willebrand factor; WHR, waist-to-hip ratio. Data are mean \pm SD. Differences between groups were analysed by twotailed unpaired Student's t-test. 
Table 3 Univariate analysis of the correlation between NAMPT circulating concentrations and mRNA NAMPT expression levels in peripheral blood cells with other variables.

\begin{tabular}{|c|c|c|c|c|}
\hline \multirow{2}{*}{$\begin{array}{l}\text { NAMPT circulating } \\
\text { concentrations }\end{array}$} & \multicolumn{2}{|c|}{ Unadjusted } & \multicolumn{2}{|c|}{ BMI Adjusted } \\
\hline & $\mathbf{r}$ & $\mathbf{P}$ & $\mathbf{r}$ & $\mathbf{P}$ \\
\hline BMI & 0.31 & 0.044 & - & \\
\hline WHR & 0.47 & 0.012 & 0.50 & 0.008 \\
\hline Glucose & 0.33 & 0.049 & 0.29 & 0.129 \\
\hline Triglycerides & 0.42 & 0.011 & 0.38 & 0.025 \\
\hline Cholesterol & 0.51 & $<0.001$ & 0.48 & 0.004 \\
\hline LDL-cholesterol & 0.53 & $<0.001$ & 0.47 & 0.006 \\
\hline C-reactive protein & 0.35 & 0.042 & 0.21 & 0.234 \\
\hline Fibrinogen & 0.33 & 0.047 & 0.16 & 0.352 \\
\hline Uric acid & 0.47 & 0.006 & 0.38 & 0.034 \\
\hline $\mathrm{ALT}$ & 0.49 & 0.002 & 0.41 & 0.013 \\
\hline AST & 0.45 & 0.005 & 0.41 & 0.014 \\
\hline$\gamma-\mathrm{GT}$ & 0.45 & 0.006 & 0.41 & 0.014 \\
\hline $\begin{array}{l}\text { PBC NAMPT mRNA } \\
\text { expression }\end{array}$ & $\mathbf{r}$ & $\mathbf{P}$ & $\mathbf{r}$ & $\mathbf{P}$ \\
\hline BMI & 0.50 & 0.009 & - & - \\
\hline Body fat & 0.48 & 0.014 & 0.11 & 0.614 \\
\hline WHR & 0.43 & 0.032 & 0.15 & 0.483 \\
\hline Insulin & 0.54 & 0.014 & 0.34 & 0.155 \\
\hline HOMA & 0.59 & 0.016 & 0.47 & 0.074 \\
\hline Triglycerides & 0.49 & 0.033 & 0.44 & 0.043 \\
\hline Fibrinogen & 0.48 & 0.039 & 0.16 & 0.513 \\
\hline vWF & 0.59 & 0.013 & 0.44 & 0.088 \\
\hline ALP & 0.52 & 0.020 & 0.49 & 0.050 \\
\hline$\gamma$-GT & 0.50 & 0.031 & 0.45 & 0.058 \\
\hline mRNA HIF1- $\alpha$ & 0.51 & 0.011 & 0.45 & 0.030 \\
\hline
\end{tabular}

ALP, alkaline phosphatase; ALT, alanine aminotransferase; AST, aspartate aminotransferase; BMI, body mass index; $\gamma$-GT, $\gamma$-glutamyltransferase; HIF1-a, hypoxia-inducible factor-1-a; HOMA, homeostatic model assessment; PBC, peripheral blood cells; vWF, von Willebrand factor; WHR, waist-to-hip ratio. Statistical significant values are in bold. 
Table 4 Multiple regression analysis with total-cholesterol, HDL-cholesterol LDLcholesterol, triglycerides and CRP as independent variables.

NAMPT circulating concentrations

\begin{tabular}{lll}
\hline $\mathrm{r}^{2}=0.356 ; \mathrm{P}=0.004$ & $\beta$ & $\mathrm{P}$ \\
\hline Total-cholesterol & 1.08 & 0.016 \\
HDL-cholesterol & -0.66 & 0.036 \\
LDL-cholesterol & -0.59 & 0.183 \\
Triglycerides & 0.37 & 0.050 \\
CRP & -0.38 & 0.135 \\
\hline
\end{tabular}

CRP, C-reactive protein; NAMPT, nicotinamide phosphoribosyltransferase.

Statistical significant values are in bold. 


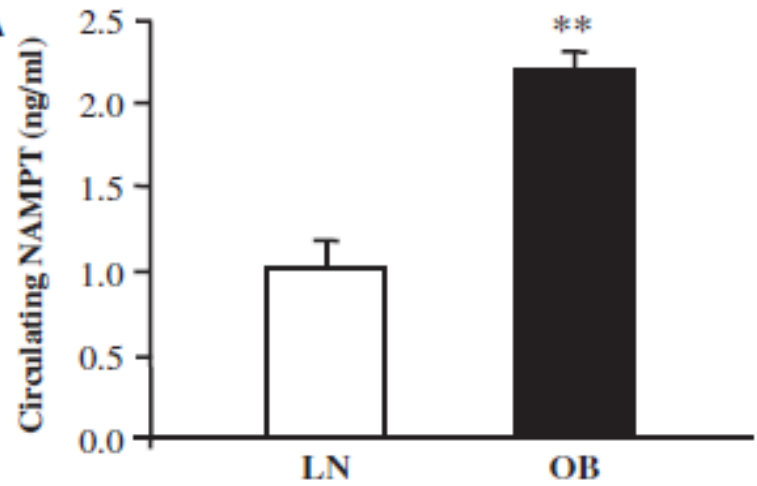

B

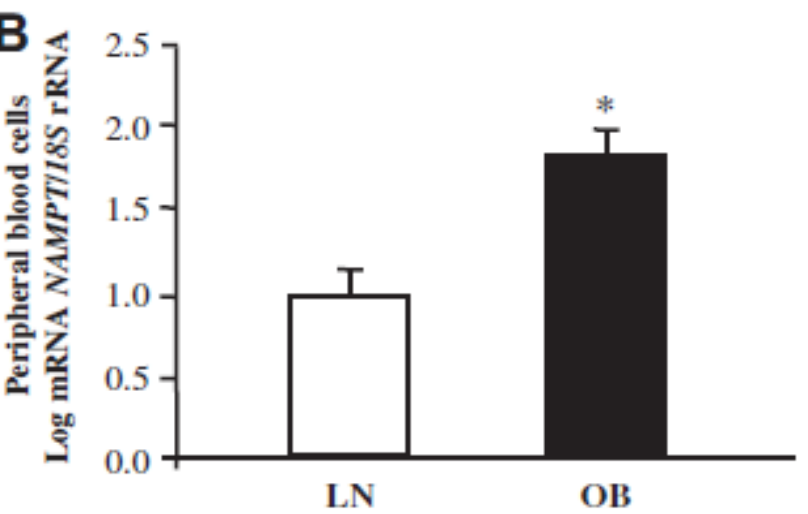

C

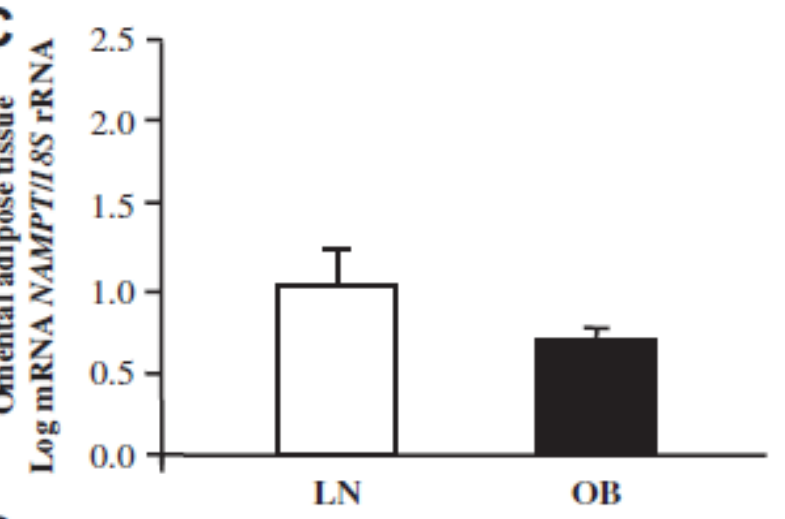

D

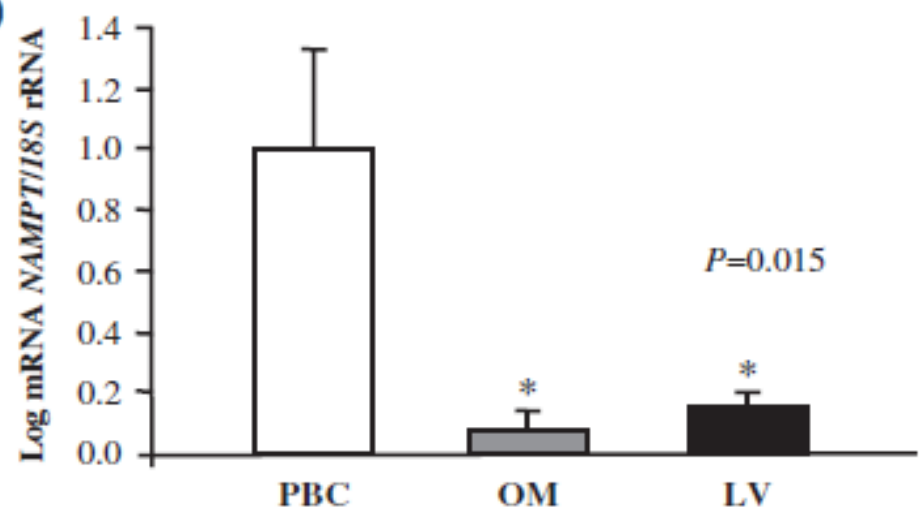

Figure 1

(A) Circulating concentrations of NAMPT of lean (LN) and obese (OB) volunteers. Bars represent the mean $f$ SD. Differences between groups were analysed by two-tailed unpaired Student's t-test. ${ }^{* *} \mathrm{P}<0.01$ vs LN. $\mathrm{LN}: \mathrm{n}=14$; OB: $\mathrm{n}=30$.

(B) Real-time PCR analysis of NAMPT transcript levels in peripheral blood cells (PBC) of lean $(\mathrm{LN})$ and obese $(\mathrm{OB})$ subjects. Bars represent the mean $\mathrm{f} S \mathrm{SD}$ of the ratio between gene expression to $18 \mathrm{~S}$ rRNA. The expression level in lean subjects was assumed to be 1 . Differences between groups were analysed by two-tailed unpaired Student's t-test. ${ }^{*} \mathrm{P}<0.05$ vs LN. LN: $\mathrm{n}=8 ; \mathrm{OB}: \mathrm{n}=18$.

(C) Real-time PCR analysis of NAMPT transcript levels in omental adipose tissue of lean (LN) and obese (OB) subjects. Bars represent the mean $\mathrm{f} \mathrm{SD}$ of the ratio between gene expression to $18 \mathrm{~S}$ rRNA. The expression level in lean subjects was assumed to be 1. Differences between groups were analysed by two-tailed unpaired Student's t-test. $\mathrm{LN}: \mathrm{n}=5$; OB: $\mathrm{n}=18$.

(D) Real-time PCR analysis of NAMPT transcript levels in peripheral blood cells (PBC), omental adipose tissue (OM) and liver (LV) of obese subjects. Bars represent the mean $\mathrm{f} \mathrm{SD}$ of the ratio between gene expression to $18 \mathrm{~S}$ rRNA. The expression level in PBC was assumed to be 1. Differences among groups were analysed using the Kruskal-Wallis test, followed by U Mann-Whitney's pairwise comparisons. $n=4$ per group. 